\title{
A Structured Approach to Evaluate Collaborative Educational Virtual Environments
}

\author{
Rosa Reis $^{1}$, Benjamin Fonseca $^{2}$, Paula Escudeiro ${ }^{1}$ \\ ${ }^{1}$ GILT- Graphics Interaction Learning Technologies Polytechnic of Instituto Superior de Engenharia do Porto, Porto, Portugal \\ ${ }^{2}$ Department of Int2INESC-TED/University of Trás-os-Montes e Alto Douro, Vila Real, Portugal \\ \{rmr\&pmo\}@isep.ipp.pt, benaf@utad.pt@
}

\begin{abstract}
In this paper we present a 3D collaborative educational virtual environment, which was development by utilizing an iterative applied throughout development cycle. The main propose was demonstrating applicability of an evaluation framework idealized by the authors. Our evaluation framework is supported by the Quantitative Evaluation Framework (QEF) proposed by Escudeiro [3]. This framework uses the standard of reference ISO 9126 (is an international standard for the evaluation of software) and measures the quality relatively to a hypothetical ideal system, whose quality is assumed to be $100 \%$. It was created with main objective to assess the educational software.

The 3D collaborative educational virtual environment was evaluated both during his development lifecycle in the laboratory and in the user's natural work environments. Our first evaluation suggests that the quality assessment must be done. The environments are created to be use by teachers and students in order to aid the teaching / learning process and in most of the cases, the environments do not have appropriate technical characteristics, and neither has characteristics related with to the area of study.
\end{abstract}

Index Terms - Software quality, Educational Collaborative Virtual Environments..

\section{Introduction}

The traditional education is no longer appealing to new students, because they can no longer stand still long to attend in a class. Given this fact, it is necessary that education keep pace with the changes. The new generation is more attracted by Technologies in order to facilitate the linkage between communication and social networking [1]. This factor has allowed the emergence of new forms of learning, emphasizing the new environments called the virtual Worlds. The virtual worlds appear as a new generation of information technology being used in the learning process. With these worlds we can create spaces which provide set of services. However most environments, particularly the collaborative virtual environments have not appropriate technical characteristics and neither has characteristics related with this study field. In this sense, when being developed, it is essential that an assessment should be made taking into account the application field where the software will be used. For that we must identify the requirements for educational collaborative virtual environments, which will help us to get a quality product. The software quality is "the compliance to requirements and the characteristics implicit that are expected in professionally developed software" [1]. The quality assessment must be done because these environments are created to be used by teachers and students in order to aid the teaching / learning process.
This paper will focus on the evaluation process the 3D collaborative education virtual environments because we believe in needs to assessment the applications during their development lifecycle and also into educational environment with the learners. In this sense is organized as follows: section 2 presents an evaluation model of educational collaborative virtual environments; section 3 presents a $3 \mathrm{D}$ collaborative educational virtual environment, which aims to prove applicability of evaluation model that guides us to get a product with quality; finally we draw some conclusions about the advantages of assessment the environments

\section{Proposed Model}

The proposed model (Fig 1) is composed by five steps. These steps include activities that allow quantifies the quality Educational Collaborative Virtual Environments (ECVE). It is based on the Quantitative Evaluation Framework (QEF) developed by Escudeiro [3]

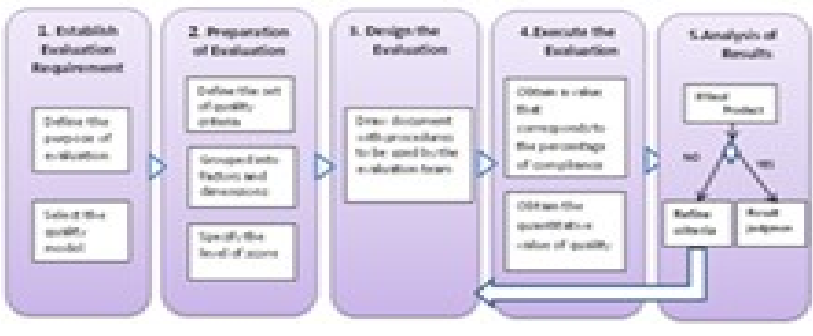

Fig. 1 Phases of Assessment Model

Below, it is possible to observe in detail each of the steps that constitute our proposed evaluation model.

\section{A. Establish the Evaluation Requirements}

Before starting a product quality assessment, it is necessary to know the purpose of evaluation related with the intended use of the product. Then, it is necessary to select the quality model. In our case, this model is the Qualitative Evaluation Framework (QEF) developed by Escudeiro in 2008 [3]. This framework evaluates the educational software quality and gives us and gives us degree of freedom to choose the criteria, which can be using in any domain and valence. It is based on the standard of reference ISO 9126 (is an international standard for the evaluation of software) and measures the quality relatively to a hypothetical ideal system, whose quality is assumed to be $100 \%$. 
We need firstly to identify a set of quality requirements, which will are grouped into factors according to theirs characteristics. Subsequently the factors will be grouped into dimensions. The next activity will be the association of weights to each requirement according to their relevance in the product, i.e., it's percentage of compliance with the criteria. Then, we need calculate the percentage of compliance of each factor. It is calculated by the following formula

$$
\text { Factor }_{n}=\sum_{n} p r_{m} \times\left(\sum_{m} p r_{m} \times p c_{m}\right)
$$

where, the $m$ is the number of relevance criteria to the factor in analysis; $\mathrm{pr}_{\mathrm{m}}$ is the weight of criteria $\mathrm{m}$ and $\mathrm{pc}_{\mathrm{m}}$ is the percentage of compliance with the criteria. Finally, we need calculated the system quality. The system quality is computed by

$$
\text { Quality }=1-D / \sqrt{n} \ldots \ldots \rightarrow D={\sqrt{\sum\left(1-D I M_{J} / 100\right)}}^{2}
$$

where D represents the global deviation (Euclidean distance between our system coordinates and the ideal system, whose coordinates is $(1,1,1)$.

\section{B. Preparation for Evaluation}

We must define the set of quality criteria for the product in analysis. The criteria define possible primitive attributes to be evaluated. The requirements define possible attributes to be evaluated. We emphasize three aspects: technical/functional, educational/didactic and usability. These aspects are intrinsically dependent, because a collaborative educational virtual environment should allow an improvement in the teaching/learning process, which is not possible to carry out using traditional methods. It must provide a high degree of interactivity, encouraging the teamwork, according to the needs and preferences giving the user the feeling of presence which promotes the learning process. They should be grouped into factors, and then into dimensions. The factors determine the quality from the different user's viewpoint of the product. The dimensions are based on ISO 9126 [2]. These factors and dimensions are designed to cover all the aspects that must be considered when evaluating the quality of the Educational Collaborative Virtual Environment. We can observe a possible aggregation of dimensions, factors and criteria, in section 3.2 .

\section{Design of evaluation}

In this phase we must draw a document with the procedures to be used by the evaluation team.

\section{Execute the evaluation}

This step corresponds to the measuring of the evaluation process as described in QEF framework Its main purpose is to provide quantitative results for the product's evaluation, which is acceptable and reliable by the evaluators. The main goal of this phase is to obtain a value that corresponds to the percentage of compliance tests performed for each requirement. After that it will be triggered an automatic calculation of the quality system by applying the formulas derived by the QEF framework. In the end, we obtain the quantitative value of quality that will be discussed during lifecycle product.

\section{E Analysis of results}

Gives rise to two substeps, which are independent. This independence is related to the type of assessment, i.e., the control for quality can be performed through in two instances: if it is a final product or a product that is under development. In the first case the evaluation processes consists only in evaluating the result obtained from the quality and to make a value judgment, i.e. analyzes the value obtained in relation to a pre-established model.

\section{The 3D Collaborative Educational Virtual Environment}

\section{A. Overview}

Our environment will help in support a math class of 5th grade of Basic Education. The topic to be addressed is related to the Geometric Solids. The target audience is children 10 to 11 years old. For his conception we used the OpenSimulator platform. OpenSimulator is an open source multi-platform where we can create complex 3D environments [4] and includes facilities for creating custom avatars, chatting with others in the environment, building 3D content in world.

The environment has a set of collaborative activities structured as the interaction of avatars with 3D objects and other avatars. They were designed to acquire skills involved in mathematics. It was divided into three main areas: information space, discussion space and learning space, as seen in figure 2. It was built through of several iterations. On each iteration of the process, was verified fulfillment the quality criteria. If the requirements were not fulfilled, the development team was making adjustments to the product and so, we advanced with the construction of system.

\section{B. Evaluation}

We assess the environment during his lifecycle development. First, we had to consider who should take part in the evaluation. An evaluator's team composed by a group of quality specialist and teachers take part of the evaluation and begin by defining a set of quality requirements. In table 1 we can see the requirements of environment.

After define the requirements, the team grouped them into factors and the factors in dimensions according with QEF. In this step some decisions has to be taken, for example: What factors and dimensions will be the most appropriate?

After collecting all information, during some brainstorming session, the team decided to take into account three dimensions: Technical/Functional, Pedagogical/Didactic and Ergonomic. We choose these dimensions because they are based on ISO 9001 and Technical/Functional dimension reflects the characteristics associated with the technical/functional aspects. This dimension covers the set of attributes that are related with the quality of the system, such as reliability, security, functionality, adaptation; the Ergonomic dimension addresses the set of scientific information needed to interactions among humans and the design of tools, machines and devices that can be used with 
maximum of comfort, safety and efficacy; and finally the Pedagogical/Didactic dimension is the property of the educational process. The requirement for collaborative learning that provides spaces in which the discussion occurs among students, when they explore concepts, seeking to combine a particular situation with social interactions that may contribute to individual and group learning.

The factors were chosen because:

Usability (Ease of use) - Any application designed should allow that all available information can be easily accessed; allowing any student (participant) is able to develop their activities.

Didactic Contents - If you consider that "knowledge must precede the action and that action must always be tied to a specific context and goals" [4], it is not difficult to understand that the educational content are key to its credibility and support learning

Learning - The learning process is a process by which information becomes knowledge. Students should learn without memorizing, create mental representations of the context in which they are involved. An ECVE should provide all Students an Opportunity to Learn through a range of activities such as simulation activities, collaboration and social experiences. This learning should be active, where students are actively involved in the learning process, becoming fully immersive. So, any ECVE should provide resources for performing tasks collaboratively, answering questions and information.

Navigation - Is a factor influencing the quality of ECVE, which depends on a combination of characteristics resulting from the environment, user, tasks, navigation strategy, aids navigation and controls of navigation.

Interaction- When speaking of collaborative learning as an activity that involves the construction of knowledge in a collective way, we can easily see that a ECVE must incorporate a set of activities to allow participants can build their knowledge model, expressing, criticizing, and sharing each element of that model.

TABLE I Results obtained in percentage in different iterations

\begin{tabular}{|c|c|c|c|c|c|c|}
\hline DI & FT & Criteria & 1 IT & 2 IT & 3 IT & 4 IT \\
\hline \multirow{8}{*}{$\begin{array}{l}\text { Technical / } \\
\text { functional }\end{array}$} & \multirow{8}{*}{ Ease of use } & The system allows you to test the existence of other avatars (participants). & 80 & 100 & 100 & 100 \\
\hline & & The environment allows a degree of realism, but the user is aware that the reality is virtual. & 50 & 70 & 80 & 90 \\
\hline & & The environment allows the user to explore and act naturally. & 60 & 80 & 100 & 100 \\
\hline & & $\begin{array}{l}\text { The effects of user actions on the virtual objects are immediately visible and are in } \\
\text { accordance with the laws of physics and perceptual user expectations. }\end{array}$ & 100 & 100 & 100 & 100 \\
\hline & & Active objects are clearly marked and explained to promote learning. & 70 & 80 & 100 & 100 \\
\hline & & The user' involvement in the environment is more natural as possible. & 100 & 100 & 100 & 100 \\
\hline & & The environment cans provide tasks, missions and rules to the users. & 50 & 70 & 90 & 100 \\
\hline & & The environment allows users to search for materials that describe the setting and content & 80 & 100 & 100 & 100 \\
\hline \multirow{9}{*}{$\begin{array}{l}\text { Pedagogica } \\
1 \text { Didactical }\end{array}$} & \multirow{6}{*}{$\begin{array}{l}\text { contents } \\
\text { didactics }\end{array}$} & The graphs and figures are consistent not causing the student' disorientation. & 80 & 100 & 100 & 100 \\
\hline & & $\begin{array}{l}\text { The content is supported by deterrents types of multimedia media ( video, sound, images, } \\
\text { etc) }\end{array}$ & 100 & 100 & 100 & 100 \\
\hline & & The metaphor is consistent with the tasks of the environment. & 100 & 100 & 100 & 100 \\
\hline & & The information in the environment is consistent with the subject matter covered. & 100 & 100 & 100 & 100 \\
\hline & & $\begin{array}{l}\text { The content and messages are not negative and tendentious and there is no discrimination } \\
\text { based on social class, religion, .... }\end{array}$ & 100 & 100 & 100 & 100 \\
\hline & & The information is well structured properly differentiating the goals, opinions, multimedia. & 100 & 100 & 100 & 100 \\
\hline & \multirow{3}{*}{ Learning } & The tasks are designed according to the profile of the user group & 50 & 100 & 100 & 100 \\
\hline & & The responses to actions are given enough time & 100 & 100 & 100 & 100 \\
\hline & & $\begin{array}{l}\text { The environment has an area for the tasks performed and should be structured, possibly } \\
\text { hierarchically in order to reflect the division of work and resources to organize by members } \\
\text { of the group }\end{array}$ & 60 & 80 & 100 & 100 \\
\hline \multirow{6}{*}{ Ergonomics } & \multirow{3}{*}{ navigation } & The environment contains a map which facilitates the visual representation. & $\mathbf{0}$ & 50 & 80 & 100 \\
\hline & & $\begin{array}{l}\text { The environment allows the user to be able to return to the previous point without } \\
\text { disorienting. }\end{array}$ & 30 & 30 & 50 & 100 \\
\hline & & The user can choose the path to move & 30 & 30 & 100 & 100 \\
\hline & \multirow{3}{*}{ Interaction } & $\begin{array}{l}\text { The environment makes available interactive tools allowing some form of communication? } \\
\text { (chat, forum, message or e-mail, communities) }\end{array}$ & 20 & 40 & 60 & 100 \\
\hline & & $\begin{array}{l}\text { The user effectively controls the performance of a series of tasks and task sequence in an } \\
\text { appropriate way to achieve these }\end{array}$ & 0 & 0 & 40 & 90 \\
\hline & & $\begin{array}{l}\text { Is given the student the opportunity to select information that allows relate important } \\
\text { information for the type of activity which is engaged }\end{array}$ & 20 & 60 & 80 & 100 \\
\hline
\end{tabular}


The requirements were then, after validation, grouped into 6 factors in order to obtain a coherent group. The factors were also grouped into three dimensions as can be seen in table 1 , with more details.

\section{Analysis of Results}

Performing a statistical analysis of the results, we obtain the graphics below, which shows us the importance of assessing the AVCEs during its development process.

We can observe (see fig. 2) that as the prototype was evolved to a final product, the quality was increasing. This is due to fulfillment of a larger number of quality requirements considered ideal for our system (our ideal system is one that fulfills all requirements in full). In the fourth iteration was not obtained a value of $100 \%$ because of how OpenSim was installed and limitations in terms of hardware of the machine that served as the workplace.

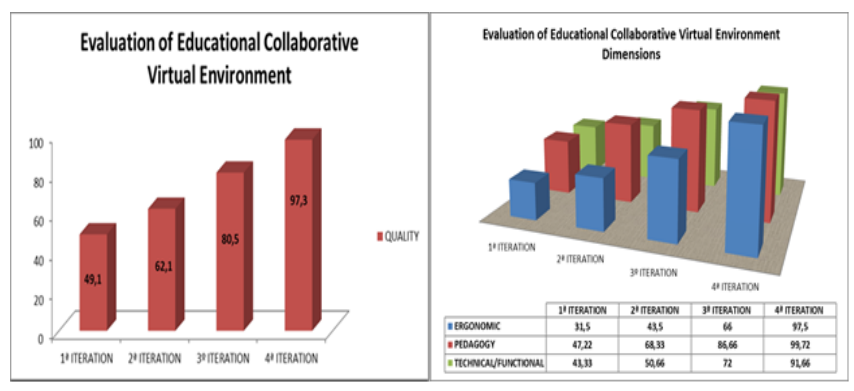

For a more accurate assessment we decided to analyze the quality of the environment in terms of its dimensions, reaching values shown in figure 2 .

The authors consider that these factors are important because they lead to changes in how the student understands the concepts (change their cognitive structures). The content and activities should be prepared with emphasis on "learning to learn" and in self-study. Finding the balance in the way of presenting content and concept to be transmitted is a big challenge because we work with stimuli that go beyond the vision.

\section{Conclusion}

This paper reports the design a 3D collaborative educational virtual environments developed from an assessment structured approach which allow us to develop and select an adequate ECVE to support the learning/teaching process. This approach aims to structure the process of assessment based on the Quantitative Evaluation Framework [3], because it allows to measure quality of the final product, and evaluate the systems quality at any moment during its lifecycle. This framework gives freedom to select the quality criteria, and allows obtaining a single quantitative value of quality for any domain in analysis. The evaluators have the responsibility in the selection of criteria, which must be relevant according to the domain under study.

However, this study still needs to be refined to highlight the benefits of ECVE evaluation, and can be introduced in a Teaching/Learning process. At moment, a focus group carries out a set of usability tests with aims to observe learners using the product to discover error and areas of improvement, for example, efficiency, accuracy, recall, and emotional response. After that, it will be integrated in work related to a European project designated of ICTWays for Science Classrooms (528103-LLP-1-2012-1-PT-COMENIUS$\mathrm{CNW}$ ) whose focus is to "promote the use of next generation networks for educational communities by providing services and educational content of interest, enhancing the infrastructure and technological equipment in existing public schools" and the Technological Plan for Education that identifies the need for training and certification of teachers for school modernization in Europe.

\section{References}

[1] ABNT, "Engenharia de software - Qualidade de produto": NBR ISO/IEC 9126-1:2003.

[2] Scalet et al, "ISO/IEC 9126 and 14598 integration aspects", The Second World Congress on Software Quality, Yokohama, Japan, 2000

[3] P. Escudeiro, "X-Tec Model and QEF Model: A case study." In T. Bastiaens \& S. Carliner ( Eds.), Proceedings of World Conference on ELearning in Corporate, Government, Healthcare, and Higher Education 2007, pp. 258-265.

[4] http://pt.wikipedia.org/wiki/OpenSimulator. 